\title{
A Generalized MPC Framework for the Design and Comparison of VSI Current Controllers
}

\author{
Galina Mirzaeva, Member, IEEE, Graham Clifford Goodwin, Fellow, IEEE, \\ Brendan P. McGrath, Member, IEEE, Carlos Teixeira, Member, IEEE, and Marco E. Rivera, Member, IEEE
}

\begin{abstract}
Model predictive control (MPC) has been widely advocated as a design strategy for many aspects of industrial electronics. The methodology has been strongly promoted by some researchers but has also attracted criticism from others. In this context, the purpose of this paper is twofold. First, we show that many existing and popular control strategies, including finite set MPC and linear controllers [proportional integral, proportional resonant (PR)], can be viewed as special cases of MPC. Second, we show that the predictive control framework allows one to embellish these classical control architectures with novel features and to design new and advanced control architectures to address various challenges posed by power electronics applications. The findings of the paper are supported by a practical example of designing of a novel form of PR controller with superior tracking performance and delay compensation, confirmed via simulation and experiments.
\end{abstract}

Index Terms-Control design, current control, dcac power converters, model predictive control (MPC), pulsewidth modulation inverters.

\section{INTRODUCTION}

$\mathbf{M}$ ODEL predictive control (MPC) has attracted attention from the power electronics research community. Its intuitive approach and simple implementation have made it an attractive alternative for control of various power electronic devices. While its early implementations focused on current [1], [2] and torque control [3] of inverters, more recently MPC has been applied to a variety of converter topologies and power electronic devices, including three phase, multiphase and matrix converters, multilevel inverters, ac/dc converters, etc. [4]-[7]. Some advantages of MPC approach include the ability to handle complex and nonlinear situations [8], [9] and to seamlessly deal with multiple design objectives [10], [11].

On the other hand, MPC has also attracted criticism from some who point to its inherent disadvantages. The disadvantages of MPC include exponential growth of associated computational

Manuscript received June 16, 2015; revised November 2, 2015 and February 12, 2016; accepted March 18, 2016. Date of publication June 8, 2016; date of current version August 9, 2016.

G. Mirzaeva and G. C. Goodwin are with the School of Electrical Engineering and Computer Science, The University of Newcastle, Callaghan, N.S.W. 2308, Australia (e-mail: galina.mirzaeva @ newcastle.edu.au; graham.goodwin@ newcastle.edu.au).

B. P. McGrath and C. Teixeira are with the School of Electrical Engineering and Computer Science, RMIT University, Melbourne, Vic. 3000, Australia (e-mail: brendan.mcgrath@ rmit.edu.au; carlos.teixeira@ rmit.edu.au)

M. E. Rivera is with Department of Industrial Technologies, University of Talca, Curico 3341717, Chile (e-mail: marcoriv @utalca.cl).

Color versions of one or more of the figures in this paper are available online at http://ieeexplore.ieee.org.

Digital Object Identifier 10.1109/TIE.2016.2578283

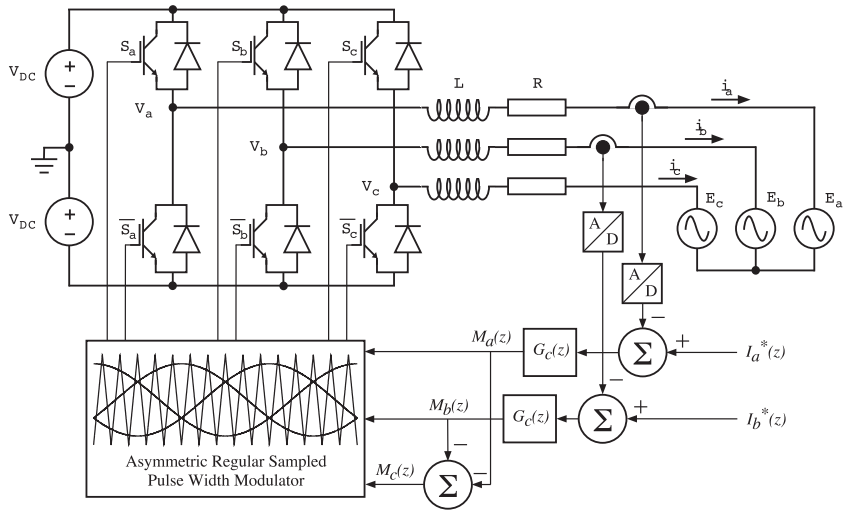

Fig. 1. VSI with PWM under closed-loop current control.

burden, reported steady-state error and stability problems [12], [13]. Another issue is the apparent disconnection between MPC and more traditional approaches, which makes it difficult to compare the performance of a vast and growing number of proposed MPC-based schemes. Some of these issues have been addressed by using more powerful microcontrollers [4], stability studies [14]-[16], and comparison studies [17], [18]. Despite a significant progress has been made, there remain open avenues for further research.

The current paper is intended to contribute to the on-going discussion relating to the application of MPC to power electronics by showing the following.

1) Many existing and popular control strategies, such as proportional integral (PI) and proportional resonant (PR), control can be given a simple and intuitive interpretation as special forms of MPC.

2) The interpretation of these controllers in the MPC framework leads to new and improved architectures for implementing these schemes.

3) Once these schemes are embedded in the MPC framework, then it becomes clear how they can be further embellished and modified to deliver enhanced properties aimed at specific design issues (e.g., delay compensation).

The ideas are general in nature and can be applied to many problems. In this study, we will consider a typical current regulation example, comprising of a three-phase voltage-source inverter (VSI) feeding a load with a back electromotive force (emf) source through a series $R L$ filter network as shown in Fig. 1. The discrete time load model for this system can be defined according to the following [19]:

$$
\left(1-a z^{-1}\right) i(z)=b z^{-1} v(z)
$$




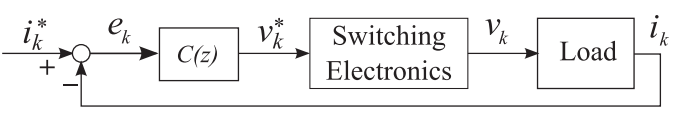

Fig. 2. Classical feedback control architecture.

where $a=e^{-\frac{\Delta}{\tau_{p}}} ; b=\frac{1}{R}\left(1-e^{-\frac{\Delta}{\tau_{p}}}\right) ; \tau_{p}=L / R$, and $\Delta$ is the sampling interval. A commonly used Euler model approximation [5] results if one only keeps the linear term of the Taylor series expansion for $e^{-x}$, namely, $a \approx 1-\Delta / \tau_{p}$ and $b \approx \Delta /\left(R \tau_{p}\right)=\Delta / L$.

This three-phase system can be represented as a pair of decoupled feedback control systems as shown in Fig. 2 where $C(z)$ is an arbitrary controller of designer's choice. In this paper, MPC horizon one approach will be applied to the design of the current controller $C(z)$ and will not be associated with a particular choice of the modulator. This choice includes pulse width modulation (PWM) or a finite set modulator.

The remainder of the paper is organized as follows. Section II revisits the antiwindup implementation of controllers with marginally stable poles. Section III presents a generalized MPC horizon one framework for optimal control design. Section IV reinterprets the existing control schemes, such as finite set MPC, and linear PI and PR controllers in an overarching MPC framework. In Section V, using the proposed framework, advanced control structures are developed, which address known stability and delay issues of the existing controllers. The advantages of the proposed advanced control structures are confirmed by simulation and experimental results in Section VI. Section VII presents conclusion and contributions.

\section{ANTIWINDUP IMPLEMENTATION}

It is well known that the implementation shown in Fig. 2 has many drawbacks. Foremost among these is the fact that, if $C(z)$ contains unstable or marginally stable poles, then the demanded voltage can "windup" due to the fact that the switching device cannot deliver at its output, $v_{k}$, the demanded voltage $v_{k}^{*}$. Actually, most popular controllers, including PI and PR, include marginally stable poles, and thus the phenomenon of windup is extremely common.

Much has been written about the problem of windup in the control literature and various solutions have been proposed [20], some of which are equivalent. A simple way of dealing with antiwindup is as described below.

Step 1. Write the reciprocal of the controller transfer function in the form $C^{-1}(z)=g_{0}+\bar{C}(z)$ where $g_{0}$ is the biproper part of $C^{-1}(z)$ while $\bar{C}(z)$ contains the dynamic section of $C^{-1}(z)$.

Step 2. Implement the controller transfer function using $g_{0}$ and $\bar{C}(z)$ within the inner feedback loop of the control system architecture as shown in Fig. 3.

It is readily seen that, if one ignores the saturation, then the transfer function from tracking error $e_{k}$ to plant input $v_{k}$ is

$$
\frac{v(z)}{e(z)}=\frac{1 / g_{0}}{1+\left(1 / g_{0}\right) \bar{C}(z)}=\frac{1}{g_{0}+\bar{C}(z)}=C(z) .
$$

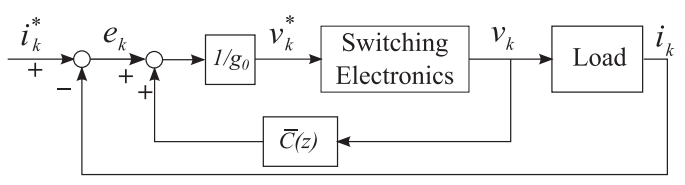

Fig. 3. Classical control architecture in antiwindup form.

Thus, in the absence of the switching device, the circuit of Fig. 3 delivers the same control as in Fig. 2. However, there is a major advantage of the implementation of Fig. 3 over that of Fig. 2, namely, all of the memory storage in the controller is captured in $\bar{C}(z)$. Moreover, in the presence of switching, saturation, etc. these memory devices are driven by the true voltage $v_{k}$ rather than the desired voltage $v_{k}^{*}$. Thus, this simple rearrangement avoids the problems of windup.

In the view of the above discussion, we will henceforth view Fig. 3 as the preferred implementation of standard classical control laws in the context of power electronics.

\section{Optimal Control Design Based on MPC HORIZON $1(\mathrm{H} 1)$}

\section{A. General Model}

A general comprehensive formulation of MPC $\mathrm{H} 1$ is adopted. Let $u_{k}$ be the input of a system and $y_{k}$ the measured output (in power electronics $u_{k}$ would, for example, be the inverter voltage and $y_{k}$ the measured output current).

We assume that the system output is corrupted by noise and periodic disturbances. We also assume that we have available a model linking $u_{k}$ to $y_{k}$. The most general form of such a model (in the linear case) can be expressed in state-space form as

$$
\begin{aligned}
x_{k+1}^{o} & =A_{o} x_{k}^{o}+B_{o} u_{k}+n_{k}^{o} \\
d_{k+1} & =A_{d} d_{k}+n_{k}^{d} \\
y_{k} & =C_{o} x_{k}^{o}+C_{d} d_{k}+m_{k}
\end{aligned}
$$

where $x_{k}^{o}, u_{k}$, and $y_{k}$ are state, input, and output vectors, respectively; $n_{k}^{o}, n_{k}^{d}$, and $m_{k}$ are Gaussian white noise sequences; $d_{k}$ is periodic disturbance; $A_{o}, A_{d}, C_{o}, C_{d}$, and $B_{o}$ are matrices of appropriate dimensions.

To describe periodic disturbances, $A_{d}$ will have eigenvalues on the unit circle. For example, a constant disturbance can be modeled by choosing $A_{d}=1$. A sinusoidal disturbance of frequency $\omega_{0}$ can be modeled by choosing

$$
A_{d}=\left[\begin{array}{cc}
0 & 1 \\
-1 & 2 \cos \omega_{0}
\end{array}\right] \text {. }
$$

The models (3)-(5) can be written in a compact form as

$$
\begin{aligned}
x_{k+1} & =\bar{A} x_{k}+\bar{B} u_{k}+w_{k} \\
y_{k} & =\bar{C} x_{k}+m_{k}
\end{aligned}
$$


where

$$
\begin{aligned}
x_{k} & =\left[\begin{array}{l}
x_{k}^{o} \\
d_{k}
\end{array}\right] ; \bar{A}=\left[\begin{array}{cc}
A_{o} & 0 \\
0 & A_{d}
\end{array}\right] ; \bar{B}=\left[\begin{array}{c}
B_{o} \\
0
\end{array}\right] ; w_{k}=\left[\begin{array}{c}
n_{k}^{o} \\
n_{k}^{d}
\end{array}\right] ; \\
\bar{C} & =\left[\begin{array}{ll}
C_{o} & C_{d}
\end{array}\right] .
\end{aligned}
$$

\section{B. Innovation Form of the Model}

We next utilize the Kalman filter to express the models (7) and (8) in the innovation form [21]. This leads to

$$
\begin{aligned}
\hat{x}_{k+1} & =\bar{A} \hat{x}_{k}+\bar{B} u_{k}+J\left(y_{k}-\bar{C} \hat{x}_{k}\right) \\
y_{k} & =\bar{C} \hat{x}_{k}+\varepsilon_{k}
\end{aligned}
$$

where $\hat{x}_{k}$ is state estimate; $\varepsilon_{k}$ is the innovation sequence provided by the Kalman observer; and the matrix $J$ defines the Kalman filter gains.

Some algebra can now be utilized to write the innovation model (9) and (10) in the transfer function form. Here and in the sequel, we use $z$ to denote a forward shift operator. This yields

$$
A(z) D(z) y_{k}=B(z) D(z) u_{k}+E(z) \varepsilon_{k}
$$

where $A(z)=\operatorname{det}\left(z I-A_{o}\right) ; D(z)=\operatorname{det}\left(z I-A_{d}\right) ; \frac{B(z)}{A(z)}=$ $C_{o}\left(z I-A_{o}\right)^{-1} B_{o}$; and $E(z)$ is a function of the matrix $J$.

Note that (11) is a known result in optimal control theory [21]. We interpret this result in the context of power electronics applications as follows: Polynomials $B(z)$ and $A(z)$ correspond to the system model without disturbance. For example, for a linear $R L$ load given by (1), $A(z)=1-a z^{-1}$ and $B(z)=b z^{-1}$. Polynomial $D(z)$ is a nulling operator for the disturbance $d$, i.e., $D(z) d=0$. For example, for any constant disturbance, $D(z)=$ $1-z^{-1}$, since $d(k)-d(k-1)=0$. Polynomial $E(z)$ depends on the choice of $J$ [21].

Let each of the polynomials in (11) be defined in the form $A(z)=a_{0}+a_{1} z^{-1}+\cdots+a_{n} z^{-n}$, etc. Then we can require that $a_{0}=1 ; c_{0}=1$, and $d_{0}=1$ without loss of generality. We also assume that the plant has $p$ units delay

$$
B(z)=z^{-p} B^{\prime}(z)
$$

where $b_{0}^{\prime} \neq 0$ and $B^{\prime}(z)$ is biproper. For example, with the load model given by (1), $B(z)=z^{-1} b$.

\section{C. p-Step Ahead Prediction}

Using the division algorithm of polynomial algebra yields

$$
\frac{E(z)}{A(z) D(z)}=F(z)+\frac{z^{-p} G(z)}{A(z) D(z)}
$$

where $F(z)=1+f_{1} z^{-1}+\cdots+f_{p-1} z^{1-p} ; G(z)=g_{0}+g_{1} z^{-1}$ $+\cdots$. Then, (11) transforms into

$$
E(z)\left(y_{k+p}-F(z) \varepsilon_{k+p}\right)=G(z) y_{k}+B^{\prime}(z) F(z) D(z) u_{k} \text {. }
$$

We notice that $F(z) \varepsilon_{k+p}$ denotes future noise, which is unpredictable at time $k$. Hence, the optimal $p$ step ahead prediction of the measured output can be written as

$$
\hat{y}_{k+p}=\frac{\alpha(z)}{E(z)} y_{k}+b_{0}^{\prime} u_{k}+\frac{\gamma(z)}{E(z)} u_{k}
$$

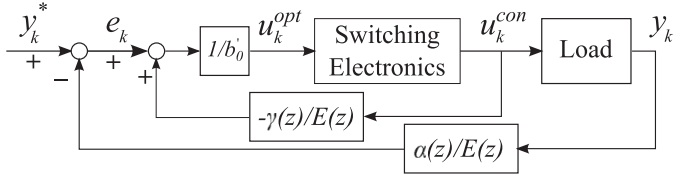

Fig. 4. MPC control architecture.

where $\alpha(z)=G(z)$; and $b_{0}^{\prime}+\frac{\gamma(z)}{E(z)}=\frac{B^{\prime}(z) F(z) D(z)}{E(z)}$.

Due to the $p$ units delay in the plant (12), the output values $y_{k+i}$ where $i<p$ will be affected only by the past control values $u_{k+i-p}$ and hence, cannot be changed by any control applied at time $k$. Therefore, even though $\hat{y}_{k+p}$ is $p$-step ahead prediction, it is only one-step ahead prediction with respect to the new control input $u_{k}$. Therefore, the presented approach still corresponds to MPC H1.

\section{Unconstrained Predictive Control Law}

We now use a standard $\mathrm{H} 1$ cost function

$$
J_{k}=\left\|y_{k}^{*}-\hat{y}_{k+p}\right\|^{2}
$$

where $y_{k}^{*}$ is the desired value of $y$; and $\|$.$\| is Euclidean norm.$

In the absence of constraints, optimizing (15) leads to the feedback control law $u_{k}=u_{k}^{\text {opt }}$ where

$$
u_{k}^{\mathrm{opt}}=\frac{1}{b_{0}}\left\{y_{k}^{*}-\frac{\alpha(z)}{E(z)} y_{k}-\frac{\gamma(z)}{E(z)} u_{k}\right\}
$$

which delivers the minimum cost function value of $J_{k}=0$.

\section{E. Constrained Predictive Control Law}

Say that we are restricted to choose $u_{k}$ from some set $U$. For example, if the switching electronics only allows a finite set of voltages, then $U$ will contain only the allowable voltages (e.g., the VSI space vector states).

In any case, it is easily seen that the optimal $p$-step ahead control policy with constraints in $U$ is $u_{k}^{\text {con }}$ where

$$
u_{k}^{\text {con }}=\left[\text { Nearest } u_{k} \in U \text { to } u_{k}^{\mathrm{opt}}\right] \text {. }
$$

This MPC control law can be represented schematically as in Fig. 4. Comparing the optimal MPC control law shown in Fig. 4 with the classical control law in antiwindup form shown in Fig. 3, one can see that the two control laws are identical with the correspondences: $g_{0}=b_{0}^{\prime} ; \bar{C}(z)=-\gamma(z) / E(z)$; $\alpha(z) / E(z)=1$. Indeed, the MPC control architecture is actually slightly more general due to the fact that we can use a transfer function in the feedback path. This may have benefits in practice, since the filter $\alpha(z) / E(z)$ allows us to filter out certain types of measurement noise.

Therefore, the proposed generalized MPC approach makes it possible to analytically compare classical control schemes with MPC-based schemes, which previously could be only compared by simulation and experiment [17]. In the proposed framework, they can be all seen as different special cases of the generalized MPC scheme.

In the sequel, to illustrate the described MPC H1 approach, we use it to design of current controllers for VSI such as to 
TABLE I

Optimal Control laws Resulting from Application of MPC H1 to Different Disturbance Models

\begin{tabular}{|c|c|}
\hline Disturbance & Optimal (unconstrained) control law to minimize the tracking error \\
\hline A) None & $v_{\mathrm{opt}}=\frac{1}{b}\left(i^{*}-a i\right)$ \\
\hline B) Const & $v_{\mathrm{opt}}=\frac{1}{b}\left(i^{*}-i\right)+\frac{(1-a) z^{-1}}{1-a z^{-1}} v$ \\
\hline C) Harmonic at $\omega_{0}$ & $v_{\mathrm{opt}}=\frac{1}{b}\left(i^{*}-\frac{a\left(1-2 \xi \cos \left(\omega_{0} \Delta\right) z^{-1}+\xi^{2} z^{-2}\right)+\varepsilon\left(1-z^{-1}\right)}{1-2 \xi \cos \left(\omega_{0} \Delta\right) z^{-1}+\xi^{2} z^{-2}+\varepsilon z^{-1}\left(1-z^{-1}\right)}\right)+\frac{\varepsilon z^{-1}\left(1-z^{-1}\right)}{1-2 \xi \cos \left(\omega_{0} \Delta\right) z^{-1}+\xi^{2} z^{-2}+\varepsilon z^{-1}\left(1-z^{-1}\right)}$ \\
\hline
\end{tabular}

TABLE II

Characteristics of the Optimal Control Laws for Different Disturbance Models

\begin{tabular}{|c|c|c|c|}
\hline Disturbance & Controller $C(z)$ & Noise sensitivity $S(z)$ & $\begin{array}{l}\text { Complementary } \\
\text { sensitivity } T(z)\end{array}$ \\
\hline A) None & $\frac{1}{b}$ & $1-a z^{-1}$ & $z^{-1}$ \\
\hline B) Constant & $\frac{1}{b} \frac{1-a z^{-1}}{1-z^{-1}}$ & $1-z^{-1}$ & $z^{-1}$ \\
\hline C) Harmonic at $\omega_{0}$ & $\frac{1}{b} \frac{1-2 \xi \cos \left(\omega_{0} \Delta\right) z^{-1}+\xi^{2} z^{-2}+\varepsilon z^{-1}\left(1-z^{-1}\right)}{1-2 \xi \cos \left(\omega_{0} \Delta\right) z^{-1}+\xi^{2} z^{-2}}$ & $\frac{\left(1-2 \xi \cos \left(\omega_{0} \Delta\right) z^{-1}+\xi^{2} z^{-2}\right)\left(1-a z^{-1}\right)}{1-2 \xi \cos \left(\omega_{0} \Delta\right) z^{-1}+\xi^{2} z^{-2}+\varepsilon z^{-1}\left(1-z^{-1}\right)}$ & $z^{-1}$ \\
\hline
\end{tabular}

minimize the tracking error corresponding to the cost function (16). Such controllers will be "optimal" in MPC H1 sense.

\section{REINTERPRETATION OF THE EXISTING CONTROL SCHEMES IN MPC FRAMEWORK}

We apply the above methodology to the $R L$ model given by (1) with disturbance, i.e.,

$$
A(z) i(z)=B(z) v(z)+d(z)
$$

where $A(z)=1-a z^{-1} ; B(z)=b z^{-1}$; and the choices for $d(z)$ include: 1) no disturbance; 2) constant disturbance; and 3) harmonic disturbance at the fundamental frequency $\omega_{0}$.

The resulting unconstrained optimal control laws are given in Table I. Each of the optimal control laws in Table I was further translated into: 1) a traditional controller form $C(z) ; 2$ ) noise sensitivity $S(z)$ which characterizes disturbance rejection; and 3 ) complementary sensitivity $T(z)$ which determines reference tracking and robustness to modeling errors. These characteristics are shown in Table II.

One interesting observation from Table I is that the gain of all the optimal controllers is equal to $\frac{1}{b} \approx \frac{L}{\Delta}$. This value and the fact that it is equal for each of the different controllers agrees with the maximum gains of PI and PR controllers found in practice by using classical methods [22].

Another observation from Table II is that, in all cases, reference is being tracked with gain 1 but with a one-step delay. This is an expected result, since in (16) we minimize the quadratic error between $y_{k}^{*}$ and $\hat{y}_{k+p}$. To avoid the delay, we may use a predicted future current reference $y_{k+p}^{*}$ instead of $y_{k}^{*}$. Alternatively, we can use a modification of the described approach, which is discussed later in Section V.

\section{A. No Disturbance: Traditional Finite Set MPC}

In the simplest case, we may ignore the disturbance by setting $d_{k}=0$ in (19). It is then easy to show that the only solution for

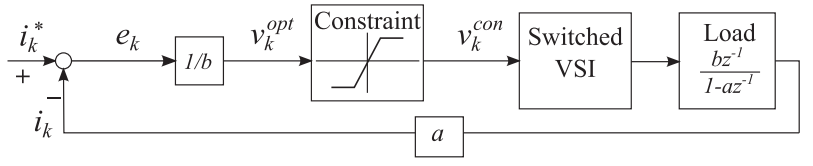

(a)

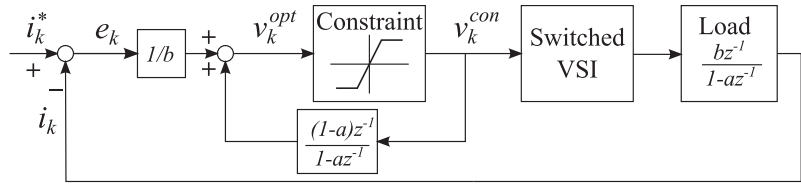

(b)

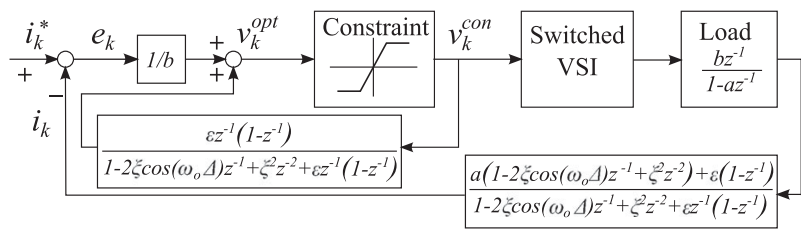

(c)

Fig. 5. Optimal controller schemes for different disturbances. (a) No disturbance: FS-MPC. (b) Constant disturbance: PI controller. (c) Harmonic disturbance: PR controller.

this case corresponds to: $\alpha(z)=a ; \beta(z)=b ; \gamma(z)=0$; and $E(z)=1$. The structures of the resulting optimal controller appear in Fig. 5(a).

If the Constraint block in Fig. 5(a) corresponds to a finite set (e.g., eight space vectors for a two-level inverter) then this case is recognizable as traditional finite set (FS) MPC H1 current control [4].

It has been shown by different researchers (see, for example, [19] and [23]) that two identical forms of FS-MPC H1 exist. Namely, one can use the prediction model (1) to evaluate the predicted current $\hat{i}$ for different voltages from the finite set, and then use the cost function $J=\left\|i^{*}-\hat{i}\right\|^{2}$. 
Alternatively, one can use the prediction model (1) to translate the reference current $i^{*}$ into the unconstrained optimal voltage $\hat{v}^{\mathrm{opt}}$, and then find the constrained optimal voltage as $\bar{v}^{\text {opt }}=\left[\right.$ Nearest $\bar{v} \in U$ to $\left.\hat{v}^{\text {opt }}\right]$ in a sense of the cost function $J=\left\|\hat{v}^{*}-v\right\|^{2}$. The latter form corresponds precisely to the optimal control solution shown in Fig. 5(a).

Such an interpretation is valid for any control scheme with a linear first-order plant. When applied to the traditional FSMPC, this interpretation explains the known drawbacks, such as parameter sensitivity and steady-state error in the presence of disturbances [12], [13]. The fundamental reason for this behavior is that the controller is optimized around the model, which does not include any disturbance.

\section{B. Constant Disturbance: PI Controller}

Typical control intervals for a VSI are in the order of $10 \mathrm{kHz}$. Over such a short interval, both reference and disturbances at the fundamental frequency can be viewed as practically constants. Therefore, by taking $d_{k}=d_{0}$ in (19) we will account not only for constant disturbances but also realistically approximate the disturbances at fundamental frequency (such as errors due to parameter mismatch, back emf, etc.).

It is easy to check that the nulling operator for a constant disturbance is $D(z)=1-z^{-1}$. There exist some freedom in the choice of $E(z)$. In practice, one would give preference to a structure with transfer function 1 in the current feedback, as shown in Fig. 5(b). This corresponds to $\alpha(z)=E(z)=1-$ $a z^{-1} ; \beta(z)=b\left(1-z^{1}\right)$ and $\gamma(z)=-b(1-a) z^{-1}$.

The resulting optimal control structure appears in Fig. 5(b). To identify this controller, we transform it into a non-anti-windup form $C(z)$ (see Table II) and then into the corresponding continuous time representation, which is given by the following:

$$
C(s)=\frac{1}{b} \frac{s-\frac{1}{\Delta} \ln a}{s}=\frac{1}{b}\left(1+\frac{1}{s \tau_{p}}\right) .
$$

It can be readily seen that the optimal controller for the case of constant disturbance is the PI controller with $K_{p}=\frac{1}{b}$ and time constant $\tau_{i}$ matching the plant time constant $\tau_{p}$. Such a controller provides zero-pole cancellation for fast dynamics and integral action for steady-state error elimination at zero frequency. Some authors intuitively arrived at the idea of including an integrator in the FS-MPC structure [13]. The presented generalized MPC approach proves that a PI controller is indeed the optimal solution for the given scenario and suggests its optimal parameters.

Also note that, in Fig. 5(b), the Constraint and Switched VSI blocks may represent, respectively, finite set constraint and zero-order hold, in which case Fig. 5(b) depicts the FS-MPC with integral action. Alternatively, these blocks may as well represent hexagonal constraint and PWM, respectively, in which case Fig. 5(b) corresponds to a linear PI controller with PWM. Thus, we have proven that the well-known linear PI control scheme is a special case of MPC with PWM and is a "close relative" of the FS-MPC with integral action.

Optimal controllers based on the constant disturbance model, although they perform better than the traditional FS-MPC, still have a limited capacity to reject the back emf disturbance, which may be desirable in drive applications [22].

\section{Harmonic Disturbance: PR Controller}

An even more realistic representation of the real inverter applications is achieved, if the disturbance and the reference are modeled as sinusoids at fundamental frequency $\omega_{0}$.

Using the nulling operator $D(z)=1-2 \xi \cos \left(\omega_{0} \Delta\right) z^{-1}+$ $\xi^{2} z^{-2}$ for the harmonic disturbance, and following the same logic as in the previous section, we obtain the optimal control structure shown in Fig. 5(c). Detailed design for this case can be found in [24]. Note three special features of this design.

First, by using $\xi<1$ we move the marginally stable system poles slightly inside the unit circle, which improves the robustness of the design with respect to digital implementation errors. Typical values of $\xi$ are 0.9-0.99. Second, in this case we can explore the existing degree of freedom denoted by $\varepsilon$ in Tables I and II and Fig. 5(c). From Table II it is clear that zero tracking error is achieved for a range of $\varepsilon$ values, however, noise sensitivity $S(z)$ can vary significantly with $\varepsilon$. Therefore, the appropriate $\varepsilon$ value can be selected from design objectives other than reference tracking. One possible value is $\varepsilon=\frac{\Delta}{\tau_{p}}$.

Third, it was not possible in this case to obtain a structure with transfer function 1 in the current feedback. The current feedback in Fig. 5(c) includes a nontrivial filter. Observe that this filter contains the same denominator function as is used for the inverter voltage feedback branch, which allows for a relatively compact practical realization.

To identify the controller in Fig. 5(c), we transform it into a non-anti-windup form $C(z)$ (see Table II) and then into the corresponding continuous time representation, is given by the following:

$$
C(s)=\frac{1}{b} \frac{s(\varepsilon / \Delta)}{(s+\varsigma)^{2}+\omega_{0}^{2}}
$$

where $\varsigma=-\frac{1}{\Delta} \ln \xi$. It is clear that $C(s)$ from (21) is a damped PR controller where $\tau_{i}=\varepsilon / \Delta$. Such a controller has proven its advantage in rejection of fundamental frequency disturbances (such as back emfs in drive applications) and is the typical choice of a linear controller when such disturbances are an issue [25]. However, the nontrivial feedback filter is a new addition to the previously known control structure.

\section{Design of Advanced Optimal Control Structures IN THE GENERALIZED MPC FRAMEWORK}

Having confirmed the power of the generalized MPC framework on the known control architectures, we can now apply MPC H1 optimal design principles to the design of advanced control structures with various desirable properties.

\section{A. Accounting for Transport Delays}

One key issue in VSI control arises due to a transport delay. Computation in a digital inverter control is typically organized as follows. At the beginning of each cycle, currents are measured; then, the voltage state or voltage sequence calculated in the 
TABLE II

Optimal CONTROL LaWs With MPC H1; Transport Delay is Accounted For

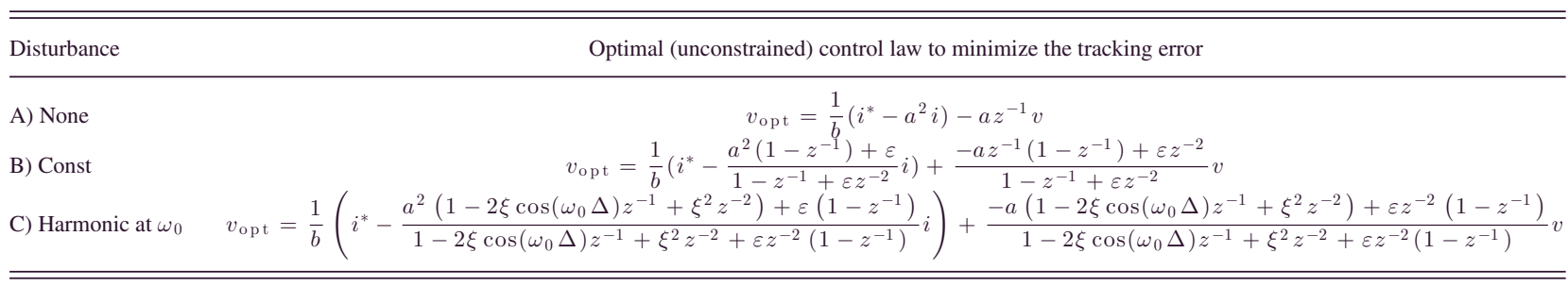

Characteristics of the Optimal Control Laws; Transport Delay is Accounted For

\begin{tabular}{lcc}
\hline \hline Disturbance & Controller $C(z)$ & $\begin{array}{c}\text { Noise sensitivity } S(z) \\
\text { Complementary } \\
\text { sensitivity } T(z)\end{array}$ \\
\hline A) None & $\frac{1}{b} \frac{1}{1+a z^{-1}}$ & $1-a^{2} z^{-2}$ \\
B) Constant & $\frac{1}{b} \frac{1-z^{-1}+\varepsilon z^{-2}}{\left(1-z^{-1}\right)\left(1+a z^{-1}\right)}$ & $\frac{\left(1-z^{-1}\right)\left(1-a^{2} z^{-2}\right)}{1-z^{-1}+\varepsilon z^{-2}}$ \\
C) Harmonic at $\omega_{0}$ & $\frac{1}{b} \frac{1-2 \xi \cos \left(\omega_{0} \Delta\right) z^{-1}+\xi^{2} z^{-2}+\varepsilon z^{-2}\left(1-z^{-1}\right)}{\left(1-2 \xi \cos \left(\omega_{0} \Delta\right) z^{-1}+\xi^{2} z^{-2}\right)\left(1+a z^{-1}\right)}$ & $\frac{\left(1-2 \xi \cos \left(\omega_{0} \Delta\right) z^{-1}+\xi^{2} z^{-2}\right)\left(1-a^{2} z^{-2}\right)}{1-2 \xi \cos \left(\omega_{0} \Delta\right) z^{-1}+\xi^{2} z^{-2}+\varepsilon z^{-2}\left(1-z^{-1}\right)}$ \\
\hline \hline
\end{tabular}

previous cycle is applied; finally, the voltage state or sequence to be applied in the next cycle is calculated.

When a new voltage is applied, its effect will be seen in the output current in a minimum of two steps: one step due to the model (19) being first order; and another step due to the transport delay. The presence of this delay is reported to cause stability problems in both traditional FS-MPC scheme [12] and high-gain linear current controllers [25]. Various stabilization strategies have been suggested in [14], [15], and [25].

In the proposed generalized MPC framework, the solution for the transport delay problem is automatic. In the first step, we account for the transport delay by including an extra delay in the actuator/plant model as

$$
\left(1-a z^{-1}\right) i(z)=b z^{-2} v(z)+d(z) .
$$

Next, exactly the same MPC-based procedure as that described in Section III is applied, with $p=2$ and $d(z)$ being the disturbance of interest. The optimal control expressions, accounting for the transport delay and rejecting the three common disturbances are summarized in Table III and their characteristics in Table IV. It is then straightforward to implement the optimal controllers from the given antiwindup forms. Note that all the controllers in Table III have nontrivial feedback filters and the complementary sensitivity $T(z)=z^{-2}$.

Consequently, they have a consistent two-cycle tracking delay between the reference and the measured output. Such a delay is usually not a problem if control is implemented at a high frequency. For low frequency and high-performance current controllers it may be desirable to avoid this delay [25]. Some researchers [12] propose to avoid the tracking delay by driving the MPC control scheme by a predicted future reference $y_{k+2}^{*}$ instead of $y_{k}^{*}$. In dynamic applications, it is not possible to predict the future reference. Another way is to compensate for the tracking delay using the MPC framework, as described below.

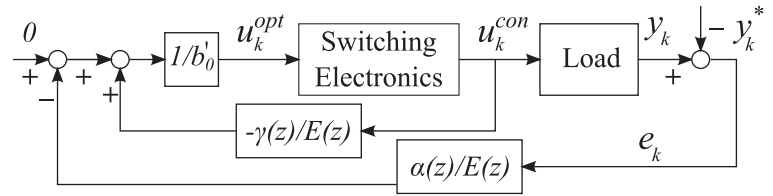

Fig. 6. Modified MPC control architecture.

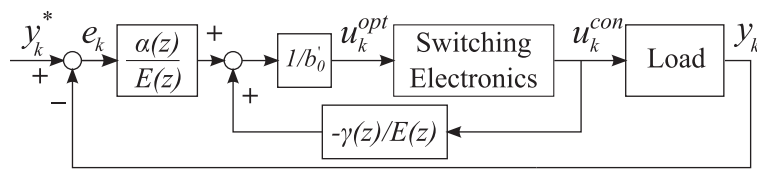

Fig. 7. MPC architecture with tracking delay compensation.

\section{B. Compensation of Tracking Delays}

Here, we propose a simple and elegant modification of the generalized MPC approach to compensate for linear tracking delays. Similar logic applies as in Section III. In addition to the model given by (3)-(5) we introduce the reference model

$$
y_{k+1}^{*}=A^{y} y_{k}^{*}+n_{k}^{y}
$$

where $A^{y}$ will have eigenvalues on the unit circle to describe periodic references; and $n_{k}^{y}$ is Gaussian white noise sequence. We extend the state vector $x_{k}$ to include vector $y_{k}^{*}$, and adjust the relevant matrices $A, B, C$, and $w$.

In Section III-B, we express $y_{k}=y_{k}^{*}+e_{k}$ where $e_{k}$ the is tracking error. Application of the Kalman observer yields

$$
A(z) D(z) e_{k}=B(z) D(z) u_{k}+E(z) \varepsilon_{k}
$$

where $D(z)$ can be interpreted as nulling operator for both disturbance and reference. In Section III-D we minimize the cost 
TABLE V

Optimal Control LaWs With MPC H1 With TRACKING Delay Compensation

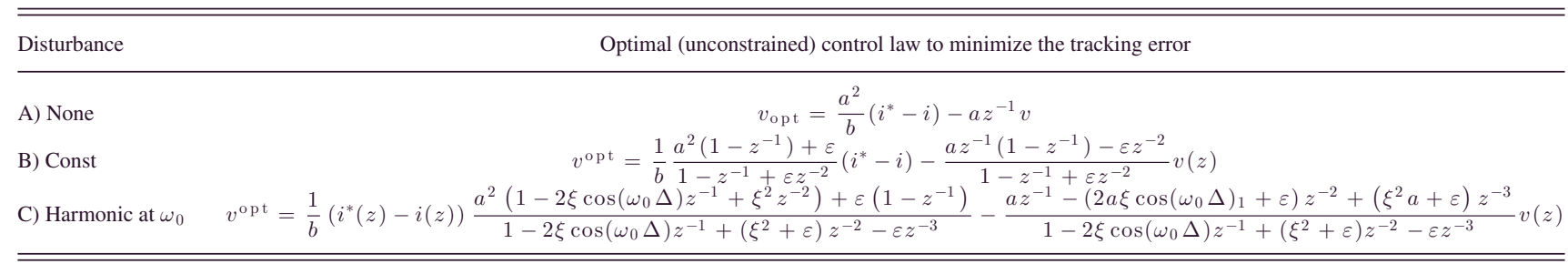

TABLE VI

Characteristics of the Optimal Control laws With Tracking Delay Compensation

\begin{tabular}{lcc}
\hline \hline & Controller $C(z)$ & Noise sensitivity $S(z)$ \\
\hline A) & $\frac{1}{b_{1} \frac{1}{1+a z^{-1}}}$ & $1-a^{2} z^{-2}$ \\
B) & $\frac{\left(1-a^{2} z^{-2}\right)\left(1-z^{-1}\right)}{1-z^{-1}+\varepsilon z^{-2}}$ & $a^{2} z^{-2}$ \\
C) $\frac{a^{2}\left(1-2 \xi \cos \left(\omega_{0} \Delta\right) z^{-1}+\xi^{2} z^{-2}\right)+\varepsilon\left(1-z^{-1}\right)}{b\left(1+a z^{-1}\right)\left(1-2 \xi \cos \left(\omega_{0} \Delta\right) z^{-1}+\xi^{2} z^{-2}\right)} \frac{\left(1-2 \xi \cos \left(\omega_{0} \Delta\right) z^{-1}+\xi^{2} z^{-2}\right)\left(1-a^{2} z^{-2}\right)}{1-2 \xi \cos \left(\omega_{0} \Delta\right) z^{-1}+\xi^{2} z^{-2}+\varepsilon z^{-2}\left(1-z^{-1}\right)}$ & $\frac{z^{-2}\left[a^{2}\left(1-2 \beta \cos \left(\omega_{0} \Delta\right) z^{-1}+\beta^{2}\left(1-z^{-1}\right)+\varepsilon\right]\right.}{1-2 \beta \cos \left(\omega_{0} \Delta\right) z^{-1}+\beta^{2} z^{-2}+\varepsilon z^{-2}\left(1-z^{-1}\right)}$ \\
\hline
\end{tabular}

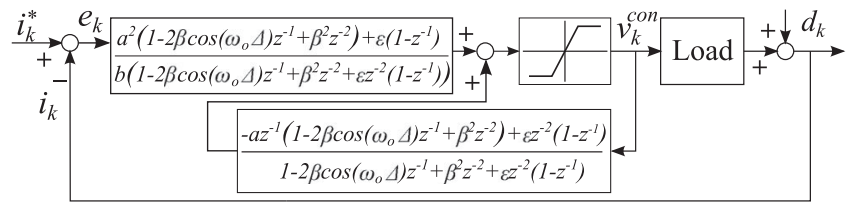

Fig. 8. MPC PR controller with tracking delay compensation.

function $J_{k}=\hat{e}_{k+p}^{2}$, resulting in unconstrained control law:

$$
u_{k}^{\mathrm{opt}}=\frac{1}{b_{0}}\left\{-\frac{\alpha(z)}{E(z)} e_{k}-\frac{\gamma(z)}{E(z)} u_{k}\right\}
$$

which delivers the minimum cost function value of $J_{k}=0$. The optimal control policy with constraints is given by $u_{k}^{\text {con }}=$ [Nearest $u_{k} \in U$ to $u_{k}^{\text {opt }}$.

The new optimal control architecture is shown in Fig. 6. Note that the main difference with the previously obtained control architecture from Fig. 4 is that the reference $y_{k}^{*}$ is subtracted at a different point. We can further rotate the diagram of Fig. 6 clockwise and invert the sign of $e$, so that the final diagram of Fig. 7 results. The feedback on measured current will always have transfer function 1 , hence no steady-state tracking delay will occur.

The expressions for the optimal controllers with respect to rejection of the three common disturbances, with the added feature of compensation for the tracking delay, are given in Table V and their characteristics in Table VI.

Based on the expressions given in Table V, we have developed an advanced control architecture with optimized reference tracking and disturbance rejection at the fundamental frequency, combined with compensation of the model and transport delays. This architecture is shown in Fig. 8.

Simulation results of Fig. 9 illustrate the transport delay compensation property of the proposed architecture in comparison with the control architecture of Fig. 5(c). A consistent two-step

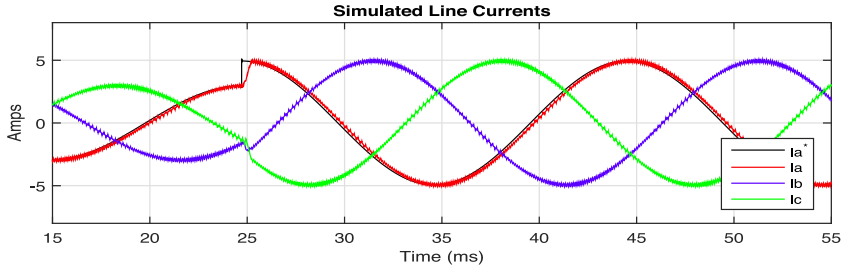

(a)

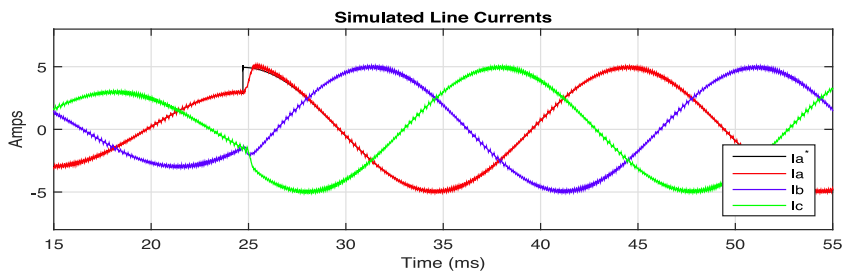

(b)

Fig. 9. Illustration of transport delay compensation. (a) Control scheme of Fig. 5(c): No delay compensation. (b) Control scheme of Fig. 8: With delay compensation.

delay between reference $i_{a}^{*}$ and actual $i_{a}$ currents, evident from Fig. 9(a), is not present in Fig. 9(b) under steady state. A further study of the proposed architecture of Fig. 8, by simulation and experiment, is presented in Section VI.

\section{Other Improvements}

1) Harmonic Cancellation: Say that, in addition to tracking the fundamental frequency $\omega_{0}$, one wishes to eliminate harmonics (say, 5th, 7th, 11th). Then, all we need to do is add these frequencies to the model $A_{d}$ or, equivalently, to the polynomial $D(z)$. Preliminary results on this functionality can be found in [26].

2) Compensation for Nonlinear Switching Delays: In addition to transport delays addressed in Section V-B, there also exist switching delays which are a function of current direction. These can be readily captured in the MPC framework 


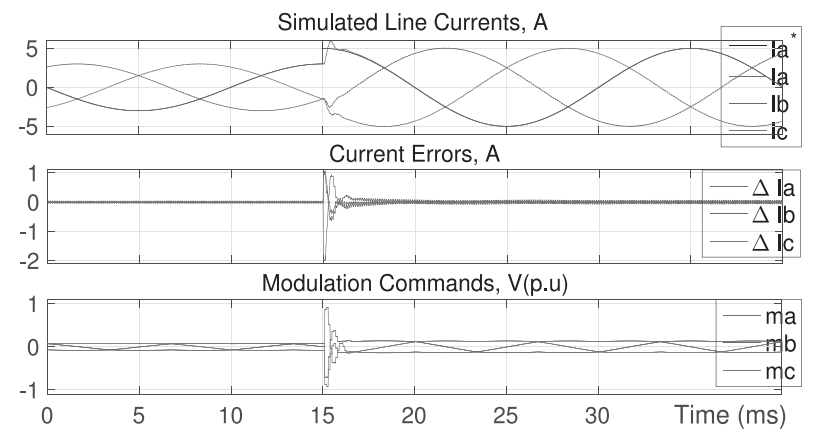

(a)

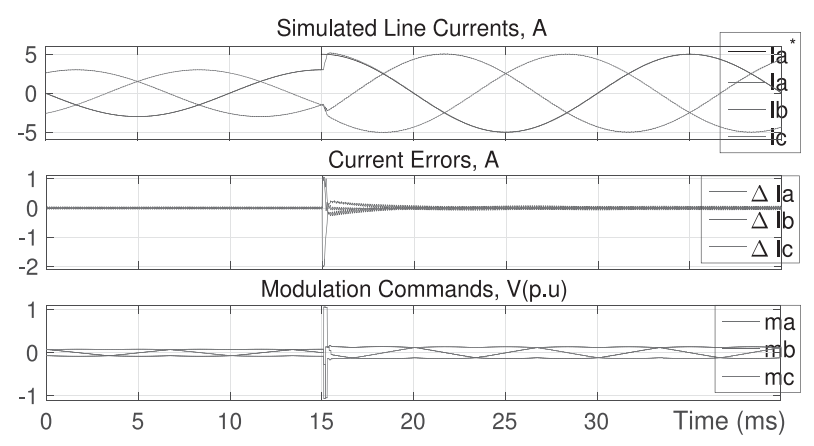

(c)

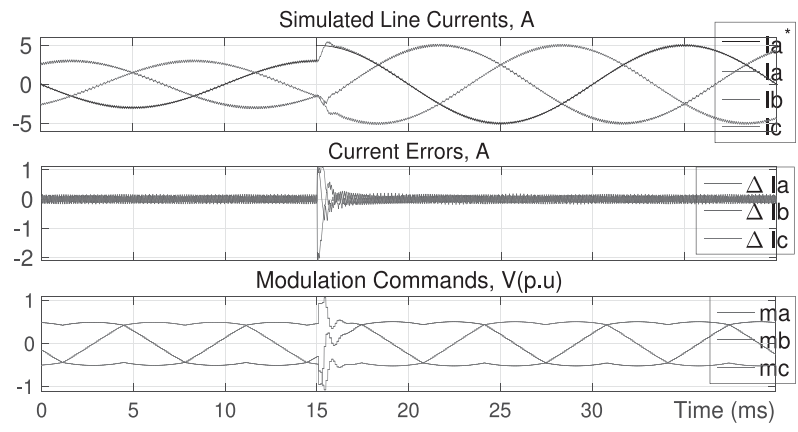

(b)

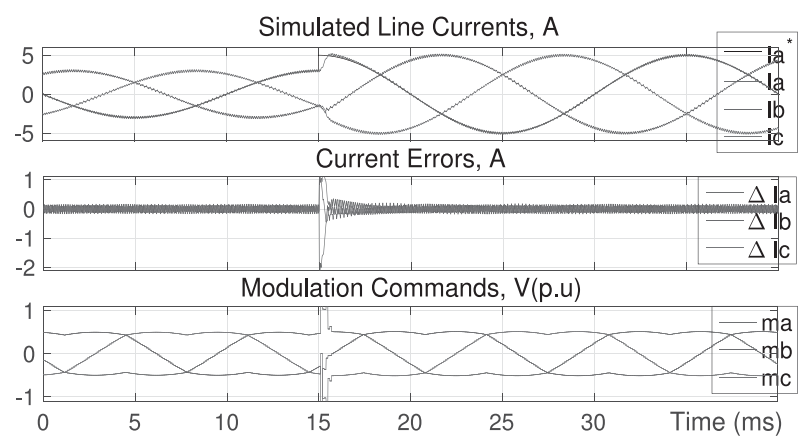

(d)

Fig. 10. PSIMC simulation results for conventional and optimal PR controllers. (a) Conventional PR: No back emf. (b) Conventional PR: With back emf. (c) Optimal PR: No back emf. (d) Optimal PR: With back emf.

by replacing the linear model (3)-(5) by a nonlinear model that accurately describes the impact of nonlinear switching delays. This is straightforward in MPC framework but impossible in the classical control (i.e., transfer function based) framework. Some results addressing the issues of nonlinear switching delays have been described in [8].

3) Alterations to the Constraint Set $U$ : It has become traditional in the contemporary MPC literature to interpret the control set $U$ as the allowable switched voltage states. However, this is by no means necessary. For example, authors of Romero et al. [27] and Vazquez et al. [28] replace the set $U$ by a finite set of switching patterns. This gives a direct link between MPC and PWM switching mechanisms.

In the case of using PWM, the average voltage can be designed to take any value within the hexagonal control set. In the context of this paper, the switching electronics block can take the form of PWM with hexagonal constraints. Two key conclusions for this embellishment are: (a) the constraint set $U$ is now convex giving vastly better computational options for optimization than FSMPC; (b) PWM can now be interpreted as a special switching mechanism in the MPC framework. This allows one to view PWM and MPC on equal footing.

\section{SimUlation AND EXPERIMENTAL REsults}

In the view of the previous remark, we have chosen to illustrate the theoretical concepts developed in this paper on an example of a current controlled VSI with a sine-triangular PWM. Such a choice sets a very high benchmark.
Current controllers for PWM VSI have been extensively researched and tuned (see [22] and [25]) leaving little room for improvement. In this paper, we intend to demonstrate that a current regulator based on the presented MPC H1 optimal structure outperforms even the best example of a current regulator of a standard structure. The use of the same sine-triangular PWM in both standard and proposed schemes will make the comparison fair and will also leave the harmonic performance outside the comparison scope.

For a standard current regulator structure we can choose either a PI controller in rotating frame or PR controller in stationary frame. Both offer very similar performance. In fact, it has been shown in [29] that a PR controller is equivalent to a PI controller transformed into a synchronously rotating frame. Therefore, the choice between PI and PR controllers is driven mostly by implementation considerations. In this paper, the authors use the PR controller scheme implemented in stationary frame for its algorithmic benefits.

Simulation models were developed in PSIM (C) software package from Powersim Inc. Simulation models and the matching experimental setup included a three-phase inverter and load with the following parameters $V_{\mathrm{DC}}=300 \mathrm{~V}, L=15 \mathrm{mH}, R=0.1 \Omega$, $f_{c}=5 \mathrm{kHz}, f_{o}=50 \mathrm{~Hz}$, and $\Delta=100 \mu \mathrm{s}$. The inverter was controlled using a TMS320F2810 fixed point DSP that was programmed to implement all inverter modulation, current regulation, protection and supervisory functions.

The advanced control architecture illustrated by Fig. 8 was simulated in PSIM alongside a conventional PR current controller. Each current controller was operated with the gains 


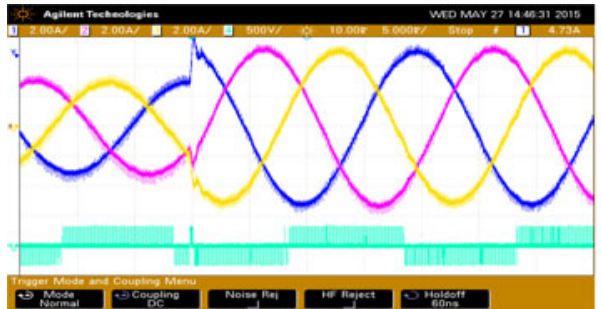

(a)

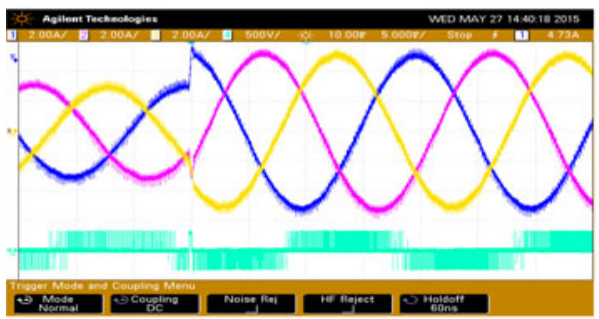

(c)

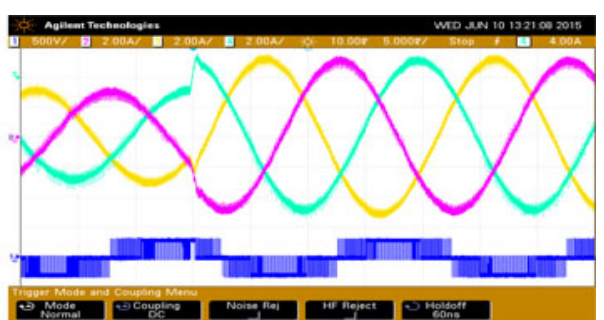

(b)

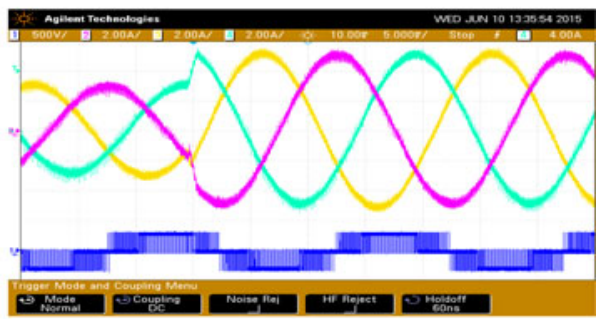

(d)

Fig. 11. Experimental results for conventional and optimal PR controllers (c) Optimal PR: No back emf. (d) Optimal PR: With back emf.

determined either through the MPC formulation detailed in this paper, or for the classical control strategies based on the principles established in [22]. The steady-state and dynamic performance of the two control structures were tested first without back emf disturbance.

The simulation results corresponding to this case are compared in Fig. 10(a) and (c). The corresponding experimental implementation when driving an L-filter without a back emf is illustrated in Fig. 11(a) and (c). The simulation and experimental results show very good agreement. Both schemes demonstrate excellent tracking performance in steady state. The transient behavior of the proposed optimal PR controller is superior to the conventional PR controller in that the transient is performed both faster and with less overshoot.

To test the rejection of the back emf disturbance, the inverter under both the classical PR and proposed MPC formulation detailed in this paper was connected to a California Instruments MX30 grid emulator, set to produce a phase voltage of $80 \mathrm{Vrms}$ at $50 \mathrm{~Hz}$. The inverter was synchronized to the MX30 using a standard second-order-generalized-integrator phase locked loop. Under these conditions the inverter is expected to clamp in an overmodulated state during transient step-change events, as the volt-drop across the filter inductance is substantially reduced.

Simulation results for both controllers under these conditions are shown in Fig. 10(b) and (d), where the longer transient compared to the case without a back emf disturbance is clearly evident. The PWM commands also clearly show the prolonged period of overmodulation for two out of the three phases. However, the response of the proposed optimal controller settles faster compared to the classical PR controller once the measured currents reach the target references. This result is also observed experimentally, see Fig. 11(b) and (d).

It is clear from these results that reference tracking of the optimal controller at least matches the classical PR controller, while transient performance is superior with less overshoot. (a) Conventional PR: No back emf. (b) Conventional PR: With back emf.

\section{CONCLUSION AND CONTRIBUTIONS}

This paper has presented a generalized MPC framework for designing VSI current controllers. It has shown that many existing controllers can be viewed as special cases of MPC. Establishing such a connection is the main contribution of this paper.

By establishing the underlying models behind the existing control schemes, this paper has given new insights into their advantages and limitations. It has shown that some performance limitations can be overcome by altering the models.

As a demonstration of the presented approach, the paper has proposed a new advanced control architecture, which combines the following features important in real world applications:

1) improved steady-state and transient reference tracking and disturbance rejection at the fundamental frequency;

2) avoiding instability issues associated with transport delay;

3) compensation of the tracking delays of both model and implementation nature.

Moreover, the concepts presented in this paper has opened the door for future embellishments including: cancellation of harmonics; compensation of nonlinear switching delays; more general modulation schemes, etc.

\section{REFERENCES}

[1] J. Rodriguez et al., "Predictive control of three-phase inverter," IET Electron. Lett., vol. 40, no. 9, pp. 561-563, Apr. 2004.

[2] A. Linder and R. Kennel, "Direct model predictive control-A new direct predictive control strategy for electrical drives," in Proc. Eur. Conf. Power Electron. Appl., Dresden, Germany, Sep. 2005, pp. 1-10.

[3] H. Miranda, P. Cortes, J. Yuz, and J. Rodriguez, "Predictive torque control of induction machines based on state-space models," IEEE Trans. Ind. Electron., vol. 56, no. 6, pp. 1916-1924, Jun. 2009.

[4] J. Rodriguez et al., "State of the art of finite control set model predictive control in power electronics," IEEE Trans. Ind. Informat., vol. 9, no. 2, pp. 1003-1016, May 2013. 
[5] S. Vazquez et al., "Model predictive control: A review of its applications in power electronics," IEEE Ind. Electron. Mag., vol. 8, no. 1, pp. 16-31, Mar. 2014.

[6] R. Ramirez, J. Espinoza, F. Villarroel, E. Maurelia, and M. Reyes, “A novel hybrid finite control set model predictive control scheme with reduced switching," IEEE Trans. Ind. Electron., vol. 61, no. 11, pp. 5912-5920, Nov. 2014.

[7] V. Yaramasu, M. Rivera, M. Narimani, B. Wu, and J. Rodriguez, "Model predictive approach for a simple and effective load voltage control of fourleg inverter with an output $l c$ filter," IEEE Trans. Ind. Electron., vol. 61, no. 10, pp. 5259-5270, Oct. 2014.

[8] G. Mirzaeva and G. Goodwin, "Harmonic suppression and delay compensation for inverters via variable horizon nonlinear model predictive control," Int. J. Control, vol. 88, pp. 1-14, Sep. 2014.

[9] S. Hoseini, J. Adabi, and A. Sheikholeslami, "Predictive modulation schemes to reduce common-mode voltage in three-phase inverters-fed AC drive systems," IET Power Electron., vol. 7, no. 4, pp. 840-849, Apr. 2014.

[10] S. Kwak and J.-C. Park, "Switching strategy based on model predictive control of VSI to obtain high efficiency and balanced loss distribution," IEEE Trans. Power Electron., vol. 29, no. 9, pp. 4551-4567, Sep. 2014.

[11] E. Fuentes, D. Kalise, J. Rodriguez, and R. Kennel, "Cascade-free predictive speed control for electrical drives," IEEE Trans. Ind. Electron., vol. 61, no. 5, pp. 2176-2184, May 2014

[12] P. Cortes, J. Rodriguez, C. Silva, and A. Flores, "Delay compensation in model predictive current control of a three-phase inverter," IEEE Trans. Ind. Electron., vol. 59, no. 2, pp. 1323-1325, Feb. 2012

[13] R. Aguilera, P. Lezana, and D. Quevedo, "Finite-control-set model predictive control with improved steady-state performance," IEEE Trans. Ind. Informat., vol. 9, no. 2, pp. 658-667, May 2013.

[14] J. Fischer, S. Gonzalez, M. Herran, M. Judewicz, and D. Carrica, "Calculation-delay tolerant predictive current controller for three-phase inverters," IEEE Trans. Ind. Informat., vol. 10, no. 1, pp. 233-242, Feb. 2014

[15] R. Aguilera and D. Quevedo, "Predictive control of power converters: Designs with guaranteed performance," IEEE Trans. Ind. Informat., vol. 11, no. 1, pp. 53-63, Feb. 2015.

[16] M. Zeilinger, M. Morari, and C. Jones, "Soft constrained model predictive control with robust stability guarantees," IEEE Trans. Automat. Control, vol. 59, no. 5, pp. 1190-1202, May 2014

[17] H. Young, M. Perez, J. Rodriguez, and H. Abu-Rub, "Assessing finitecontrol-set model predictive control: A comparison with a linear current controller in two-level voltage source inverters," IEEE Ind. Electron. Mag., vol. 8 , no. 1 , pp. 44-52, Mar. 2014.

[18] C. Lim, E. Levi, M. Jones, N. Rahim, and W. Hew, "FCS-MPC-based current control of a five-phase induction motor and its comparison with PI-PWM control," IEEE Trans. Ind. Electron., vol. 61, no. 1, pp. 149-163, Jan. 2014.

[19] G. Mirzaeva, G. Goodwin, and B. McGrath, "A new understanding and improvements of finite set model predictive control in inverter applications," in Proc. 17th Eur. Conf. Power Electron. Appl, Geneva, Switzerland, Sep. 2015, pp. $1-10$.

[20] G. Goodwin, S. Graebe, and M. Salgado, Control System Design. Upper Saddle River, NJ, USA: Prentice-Hall, 2001

[21] G. Goodwin, D. S. Carrasco, and M. M. Seron, "Predictive control: a historical perspective," Int. J. Robust Nonlinear Control, vol. 22, pp. 12961313, Aug. 2012.

[22] D. Holmes, T. Lipo, B. McGrath, and W. Kong, "Optimized design of stationary frame three phase ac current regulators," IEEE Trans. Ind. Electron., vol. 24, no. 11, pp. 2417-2425, Nov. 2009.

[23] T. Geyer and D. Quevedo, "Multistep finite control set model predictive control for power electronics," IEEE Trans. Power Electron., vol. 29, no. 12 , pp. 6836-6846, Dec. 2014

[24] G. Mirzaeva, G. Goodwin, and B. McGrath, "Optimal design of VSI current controllers based on MPC approach," in Proc. Int. Symp. Ind. Electron., Buzios, Brazil, Jun. 2015, pp. 1-6.

[25] B. McGrath, S. Parker, and D. Holmes, "High-performance current regulation for low-pulse-ratio inverters," IEEE Trans. Ind. Appl., vol. 49, no. 1, pp. 149-158, Jan./Feb. 2013.

[26] G. Mirzaeva and G. Goodwin, “Achieving perfect tracking in presence of saturation and model uncertainty in current regulators for voltage source inverters," in Proc. 1st Conf. Modelling, Identification Control Nonlinear Syst., St Petersburg, Russia, Jun. 2015, pp. 1-6.

[27] M. Romero, M. Seron, and G. Goodwin, "A combined MPC-SVM strategy for direct torque and flux control of induction motors," in Proc. 37th Annu. Conf. IEEE Ind. Electron. Soc., Melbourne, Australia, Nov. 2011, pp. $1674-1679$

[28] S. Vazquez, A. Marquez, R. Aguilera, D. Quevedo, J. Leon, and L. Franquelo, "Predictive optimal switching sequence direct power control for grid-connected power converters," IEEE Trans. Ind. Electron., vol. 62, no. 4, pp. 2010-2020, Apr. 2015.

[29] D. Zmood, D. Holmes, and G. Bode, "Frequency-domain analysis of threephase linear current regulators," IEEE Trans. Ind. Appl., vol. 37, no. 2, pp. 601-610, Mar./Apr. 2001.

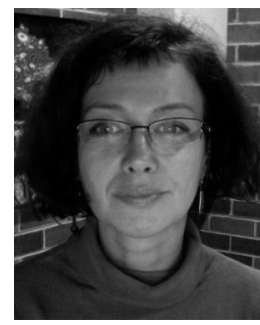

electronics.

Dr. Mirzaeva served as the Chair of the IEEE Industry Applications Society Mining Industry Committee from 2009 to 2011. Since 2014, she has been an Associate Editor of the IEEE TRANSACTIONS ON ENERGY CONVERSION.

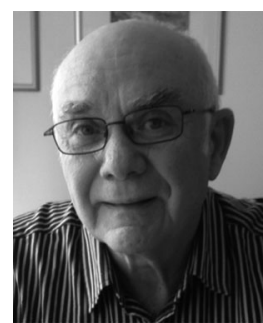

Graham Clifford Goodwin (F'86) received the B.Sc. degree in physics, the B.E. (Electrical) degree, and the Ph.D. degree in electrical engineering from the University of New South Wales, Sydney, Australia.

He is a Distinguished Professor at Harbin Institute of Technology, Harbin, China; Northwestern University, Xi'an, China; Zhengzhou University, Zhengzhou, China; and the Universidad Tecnica Federico Santa Maria, Valparaíso, Chile. He holds Honorary Doctorates from Lund Institute of Technology, Sweden, and the Technion-Israel Institute of Technology, Israel. He is the coauthor of ten books, four edited books, and several hundred papers.

Dr. Goodwin received the IEEE Control Systems Field Award in 2010 and the Rufus T. Oldenburger Medal from the American Society of Mechanical Engineers in 2013. He also received the International Federation of Automatic Control Best Engineering Text Book Award twice. He is an Honorary Fellow of the Institute of Engineers, Australia; a Fellow of the International Federation of Automatic Control; a Fellow of the Australian Academy of Science; a Fellow of the Australian Academy of Technology, Science and Engineering; a Member of the International Statistical Institute; a Fellow of the Royal Society, London, U.K.; and a Foreign Member of the Royal Swedish Academy of Sciences.

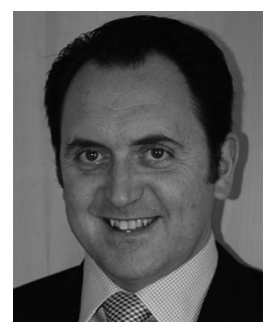

Brendan P. McGrath (M'99) received the B.E. degree in electrical and computer systems engineering in 1997, the B.Sc. degree in applied mathematics and physics in 1997, and the Ph.D. degree in electrical engineering in 2003 all from Monash University, Clayton, Vic, Australia.

He was with Monash University, the University of Newcastle, Australia, and the Laboratoire d'Electrotechnique et d'Electronique Industrielle, Toulouse, France. He is currently working at the School of Electrical and Computer Engineering, RMIT University, Melbourne, Australia. His research interests include the modulation and control of power electronic converters, with a particular emphasis on multilevel conversion systems. He has published more than 90 journal and conference articles.

Dr. McGrath received the Douglas Lampard Research Medal from Monash University for his Ph.D. thesis in 2004. He is a Member of the IEEE Power Electronics, IEEE Industry Applications and IEEE Industrial Electronics Societies, and is an Associate Editor for the IEEE TRANSACTIONS ON INDUSTRY APPLICATIONS. 


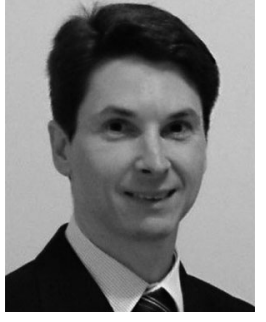

Carlos Teixeira (S'11-M'14) received the B.S. and M.S. degrees in electrical engineering from Santa Catarina State University, Joinville, Brazil, in 2000 and 2008, respectively, and the Ph.D. in electronics engineering in electrical and computer engineering from RMIT University, Melbourne, Australia, in 2014

$\mathrm{He}$ is currently with the School of Electrical and Computer Engineering, RMIT University. His research interests include the modulation and control of power electronic converters and the modeling and control of discrete-event systems. He has published more than 20 conference and journal articles and has coauthored two international patents.

Dr. Teixeira received the Statewide Academic Merit Prize from the Santa Catarina Science and Technology Foundation in 1999, and in 2000 , his outstanding undergraduate performance was recognized by the Center of Engineers and Architects of Joinville and the Regional Council of Engineers and Architects of Santa Catarina.

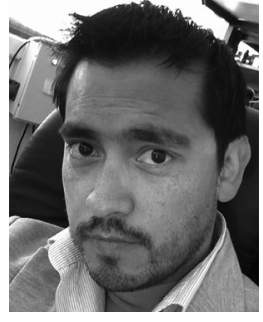

Marco E. Rivera (S'09-M'11) was born in Talca, Chile, in 1982. He received the B.Sc. and M.Sc. degrees in electrical engineering from the Universidad de Concepción, Concepción, Chile, in 2007 and 2008, respectively, and the Ph.D. degree in electronics engineering from the Department of Electronics Engineering, Universidad Tecnica Federico Santa Maria, Valparaiso, Chile, in 2011.

$\mathrm{He}$ is currently an Associate Professor with the Department of Industrial Technologies, the Universidad de Talca, Curico, Chile. His main research interests include digital control applied to power electronics, matrix converters, predictive control, and control of power converters for renewable energy applications.

Prof. Rivera received the Best Ph.D. Thesis Award from the Chilean Academy of Science in 2012, the Outstanding Engineer Award from the IEEE Chile Section in 2015, and the Second Prize Paper Award from the IEEE JOURNAL OF EMERGing AND SELECTEd TOPICS IN POWER ElECTRONICS in 2015. 